\title{
An efficient method for solving fractional Ricatti equations
}

\author{
M. Motawi Khashan ${ }^{1}$ and Muhammed I. Syam²*
}

"Correspondence:

m.syam@uaeu.ac.ae

${ }^{2}$ Department of Mathematical

Sciences, UAE University, Al-Ain,

United Arab Emirates

Full list of author information is

available at the end of the article

\begin{abstract}
In this article, we present a method to approximate the solution of a fractional Ricatti equation based on the ABFD in the Caputo sense. The proposed method depends on the fractional operational matrix of the fractional derivative. We present two examples. The results show the agreement between the exact and the approximate solutions with different choices of $\gamma$. Form the numerical results; we see that the proposed method give accurate results.
\end{abstract}

Keywords: ABFD; Fractional Ricatti equation; Fractional Legendre functions

\section{Introduction}

Fractional calculus is used to describe several problems in biology, physics, engineering, and applied mathematics. Fractional derivatives are investigated in two major ways: as lo$\mathrm{cal}$ and nonlocal derivatives. There are several definitions for the local derivatives. These definitions are considered as a direct generalization of the ordinary derivatives; see [1,2]. However, these definitions do not consider the history of the functions during computing the derivative. Other definitions are the nonlocal fractional derivatives such as the Caputo and ABFD definitions; see [3, 4]. The fractional Ricatti equation (FRE) is investigated based on different definitions of fractional derivatives. For example, see [5-7]. Akgul, et al., discussed several fractional problems using different methods such as the reproducing kernel Hilbert space method [8-16], while Alquran, et al., used a fractional power series method [17-24]. In this article, we consider the following class of equations:

$$
\begin{aligned}
& \mathfrak{f}_{\gamma}(u(x))+\mu(x) u(x)+v(x) u^{2}(x)=\xi(x), x \in(0,1), \quad 0<\gamma \leq 1, \\
& u(0)=\alpha,
\end{aligned}
$$

where $u \in H^{1}(0,1), \mu, v, \xi$ are continuous functions on [0,1] We organize our paper as follows. In Sect. 2, we present the preliminaries which we used in this paper. Method of the solution is given in Sect. 3. Some numerical results are presented in Sect. 4 to show the efficiency of the proposed method. Finally, we draw some conclusions and closing remarks.

\section{Preliminaries}

We present the basic definitions and theorems which we use in this article.

(c) The Author(s) 2019. This article is distributed under the terms of the Creative Commons Attribution 4.0 International License (http://creativecommons.org/licenses/by/4.0/), which permits unrestricted use, distribution, and reproduction in any medium, provided you give appropriate credit to the original author(s) and the source, provide a link to the Creative Commons license, and indicate if changes were made. 
Definition 1 ([4]) Let $u \in H^{1}(0,1)=\left\{u \in L^{2}(0,1): u^{\prime} \in L^{2}(0,1)\right\}$ and $0<\gamma \leq 1$. The ABFD in the Caputo sense of $u$ of order $\gamma$ is defined by

$$
£_{\gamma}(u(x))=\frac{B(\gamma)}{1-\gamma} \int_{0}^{x} u^{\prime}(s) E_{\gamma}\left(-\gamma \frac{(x-s)^{\gamma}}{1-\gamma}\right) d s
$$

For simplicity, we choose $B(\gamma)=1$. The fractional integral is defined as follows.

Definition 2 ([4]) The fractional integral is given by

$$
I^{\gamma} u(x)=\frac{1-\gamma}{B(\gamma)} u(x)+\frac{\gamma}{B(\gamma) \Gamma(\gamma)} \int_{0}^{x} u(s)(x-s)^{\gamma-1} d s
$$

Theorem 3 Let $u \in H^{1}(0,1)$ such that $£(u)$ exists. Then

$$
I^{\gamma}\left(£_{\gamma}(u(x))\right)=u(x)-u(0) .
$$

Proof See [2].

The fractional Legendre polynomials are $\left\{F L_{r}(x): r=0,1,2, \ldots\right\}$ which are defined by

$$
F L_{r}(x)=L_{r}\left(2 x^{\gamma}-1\right), \quad r=0,1,2, \ldots,
$$

where $\left\{L_{r}(x): r=0,1,2, \ldots\right\}$ are the Legendre polynomials. Then

$$
\int_{0}^{1} F L_{r}(x) F L_{s}(x) w(x) d x= \begin{cases}\frac{1}{(2 r+1) \gamma}, & r=s \\ 0, & r \neq s\end{cases}
$$

where $w(x)=x^{\gamma-1}$. Also, $F L_{r}(x)$ is given as

$$
F L_{r}(x)=\sum_{j=0}^{r}(-1)^{j+r} \frac{(r+j) !}{(r-j) !} \frac{x^{j \gamma}}{(j !)^{2}} .
$$

Theorem 4 Let $g \in C^{1}[0,1]$. Then

$$
g(x)=\sum_{r=0}^{\infty} g_{r} F L_{r}(x)
$$

where

$$
g_{r}=(2 r+1) \gamma \int_{0}^{1} g(x) F L_{r}(x) w(x) d x
$$

For the proof of these properties, see [6].

Theorem $5 I^{\gamma} F L_{r}(x)$ is a linear combination of $\left\{F L_{0}(x), F L_{1}(x), \ldots, F L_{r+1}(x)\right\}$. 
Proof Simple calculations imply that

$$
\begin{aligned}
I^{\gamma} F L_{r}(x) & =\frac{1-\gamma}{B(\gamma)} F L_{r}(x)+\frac{\gamma}{B(\gamma) \Gamma(\gamma)} \int_{0}^{x} F L_{r}(s)(x-s)^{\gamma-1} d s \\
& =\frac{1-\gamma}{B(\gamma)} F L_{r}(x)+\frac{\gamma}{B(\gamma) \Gamma(\gamma)} \int_{0}^{x} \sum_{j=0}^{r}(-1)^{j+r} \frac{(r+j) !}{(r-j) !} \frac{s^{j \gamma}}{(j !)^{2}}(x-s)^{\gamma-1} d s \\
& =\frac{1-\gamma}{B(\gamma)} F L_{r}(x)+\frac{\gamma}{B(\gamma) \Gamma(\gamma)} \sum_{j=0}^{r}(-1)^{j+r} \frac{(r+j) !}{(r-j) !} \frac{1}{(j !)^{2}} \int_{0}^{x} s^{j \gamma}(x-s)^{\gamma-1} d s \\
& =\frac{1-\gamma}{B(\gamma)} F L_{r}(x)+\frac{\gamma}{B(\gamma)} \sum_{j=0}^{r}(-1)^{j+r} \frac{(r+j) !}{(r-j) !} \frac{1}{(j !)^{2}} \frac{\Gamma(1+j \gamma)}{\Gamma(1+\gamma(1+j))} x^{(j+1) r} .
\end{aligned}
$$

Thus, $I^{\gamma} F L_{r}(x)$ is a linear combination of $\left\{F L_{0}(x), F L_{1}(x), \ldots, F L_{r+1}(x)\right\}$.

\section{Method of solution}

We want to investigate the operational matrix of $I^{\gamma}$. Define the set of $k$ block pulse functions on $[0,1)$ by

$$
\left\{p_{0}(x), p_{1}(x), \ldots, p_{k-1}(x)\right\}
$$

where

$$
p_{r}(x)=\left\{\begin{array}{ll}
1, & \frac{r}{k} \leq x<\frac{r+1}{k}, \\
0, & x \in[0,1)-\left[\frac{r}{k}, \frac{r+1}{k}\right),
\end{array} \quad r=0,1, \ldots, k-1 .\right.
$$

Then

$$
p_{r}(x) p_{s}(x)= \begin{cases}p_{r}(x), & r=s \\ 0, & r \neq s\end{cases}
$$

and

$$
\int_{0}^{1} p_{r}(x) p_{s}(x) d x= \begin{cases}\frac{1}{k}, & r=s \\ 0, & r \neq s\end{cases}
$$

where $0 \leq r, s \leq k-1$. If $g \in L_{2}[0,1]$, then

$$
g(x)=\sum_{r=0}^{k-1} g_{k} p_{r}(x)
$$

Multiply both sides by $p_{s}(x)$ then integrate from 0 to 1 to get

$$
\begin{aligned}
\int_{0}^{1} g(x) p_{s}(x) d x & =\sum_{r=0}^{k-1} g_{r} \int_{0}^{1} p_{r}(x) p_{s}(x) d x \\
& =\frac{g_{s}}{k}
\end{aligned}
$$


which implies that

$$
g_{s}=k \int_{0}^{1} g(x) p_{s}(x) d x
$$

Thus,

$$
g(x)=G^{T} P(x)
$$

where

$$
G=k\left(\begin{array}{c}
\int_{0}^{1} g(x) p_{0}(x) d x \\
\int_{0}^{1} g(x) p_{1}(x) d x \\
\vdots \\
\int_{0}^{1} g(x) p_{k-1}(x) d x
\end{array}\right), \quad p=\left(\begin{array}{c}
p_{0}(x) \\
p_{1}(x) \\
\vdots \\
p_{k-1}(x)
\end{array}\right)
$$

Theorem $6 I^{\gamma} P=\Omega P$ where

$$
\Omega=\left(\begin{array}{ccccc}
a & b & b & \cdots & b \\
b & a & b & \cdots & b \\
\vdots & \ddots & \ddots & \ddots & \vdots \\
b & b & b & \cdots & a
\end{array}\right)
$$

where

$$
a=\frac{1-\gamma}{B(\gamma)}+\frac{1}{B(\gamma) \Gamma(\gamma) k^{\gamma}}, \quad b=\frac{1}{B(\gamma) \Gamma(\gamma) k^{\gamma}}
$$

Proof For any $0 \leq r \leq k-1$

$$
\begin{aligned}
I^{\gamma} p_{r}(x) & =\frac{1-\gamma}{B(\gamma)} p_{r}(x)+\frac{\gamma}{B(\gamma) \Gamma(\gamma)} \int_{0}^{x} p_{r}(s)(x-s)^{\gamma-1} d s \\
& =\frac{1-\gamma}{B(\gamma)} p_{r}(x)+\frac{\gamma}{B(\gamma) \Gamma(\gamma)} \int_{\frac{r}{k}}^{\frac{r+1}{k}}\left(\frac{r+1}{k}-s\right)^{\gamma-1} d s \\
& =\frac{1-\gamma}{B(\gamma)} p_{r}(x)+\frac{1}{B(\gamma) \Gamma(\gamma) k^{\gamma}} \\
& =\frac{1-\gamma}{B(\gamma)} p_{r}(x)+\frac{1}{B(\gamma) \Gamma(\gamma) k^{\gamma}} \sum_{r=0}^{k-1} p_{r}(x) .
\end{aligned}
$$

Thus,

$$
I^{\gamma} P=\Omega P,
$$


where

$$
\Omega=\left(\begin{array}{ccccc}
a & b & b & \cdots & b \\
b & a & b & \cdots & b \\
\vdots & \ddots & \ddots & \ddots & \vdots \\
a & a & a & \cdots & a
\end{array}\right)
$$

and

$$
a=\frac{1-\gamma}{B(\gamma)}+\frac{1}{B(\gamma) \Gamma(\gamma) k^{\gamma}}, \quad b=\frac{1}{B(\gamma) \Gamma(\gamma) k^{\gamma}} .
$$

\section{Theorem 7 Let}

$$
F L(x)=\left(\begin{array}{c}
F L_{0}(x) \\
F L_{1}(x) \\
\vdots \\
F L_{k-1}(x)
\end{array}\right) .
$$

Then

$$
F L(x)=F P P(x),
$$

where FP is a $k \times k$ matrix with

$$
(F P)_{r, s}=\sum_{j=0}^{r}(-1)^{j+r} \frac{(r+j) !}{(r-j) !} \frac{1}{(j !)^{2}}\left(\frac{(s+1)^{j \gamma+1}-s^{j \gamma+1}}{(j \gamma+1) k^{j \gamma}}\right) .
$$

Proof For any $r \in\{0,1, \ldots, k-1\}, F L_{r}(x) \in L_{2}[0,1]$. Thus from Eq. (14), we get

$$
F L_{r}(x)=\sum_{s=0}^{k-1}(F P)_{r, s} p_{s}(x) .
$$

Hence, from Eq. (15), we get

$$
\begin{aligned}
(F P)_{r, s} & =k \int_{0}^{1} F L_{r}(x) p_{s}(x) d x \\
& =k \sum_{j=0}^{r}(-1)^{j+r} \frac{(r+j) !}{(r-j) !} \frac{1}{(j !)^{2}} \int_{\frac{s}{k}}^{\frac{s+1}{k}} x^{j \gamma} d x \\
& =\sum_{j=0}^{r}(-1)^{j+r} \frac{(r+j) !}{(r-j) !} \frac{1}{(j !)^{2}}\left(\frac{(s+1)^{j \gamma+1}-s^{j \gamma+1}}{(j \gamma+1) k^{j \gamma}}\right) .
\end{aligned}
$$

Theorem 8 FP is an invertible matrix. 
Proof From Theorem 7,

$$
F L(x)(F L(x))^{T}=F P P(x)(P(x))^{T} F P^{T} .
$$

Hence,

$$
\int_{0}^{1} F L(x)(F L(x))^{T} x^{\gamma-1} d x=F P \int_{0}^{1} P(x)(P(x))^{T} x^{\gamma-1} d x F P^{T}
$$

From Eqs. (6) and (12), we have

$$
\begin{aligned}
\int_{0}^{1} F L(x)(F L(x))^{T} x^{\gamma-1} d x & =\frac{1}{\gamma}\left(\begin{array}{cccc}
1 & 0 & \cdots & 0 \\
0 & \frac{1}{3} & \ddots & \vdots \\
\vdots & \ddots & \ddots & 0 \\
0 & \cdots & 0 & \frac{1}{2 k-1}
\end{array}\right) \\
& =\Lambda_{1}
\end{aligned}
$$

and

$$
\begin{aligned}
\int_{0}^{1} P(x)(P(x))^{T} x^{\gamma-1} d x & =\frac{1}{\gamma k^{\gamma}}\left(\begin{array}{cccc}
1 & 0 & \cdots & 0 \\
0 & 2^{\gamma}-1 & \ddots & \vdots \\
\vdots & \ddots & \ddots & 0 \\
0 & \cdots & 0 & k^{\gamma}-(k-1)^{\gamma}
\end{array}\right) \\
& =\Lambda_{2} .
\end{aligned}
$$

Therefore,

$$
\Lambda_{1}=F P \Lambda_{2} F P^{T}
$$

Thus,

$$
\operatorname{det}(F P)=\sqrt{\frac{\prod_{r=0}^{k-1} \frac{1}{\gamma(2 r+1)}}{\prod_{r=0}^{k-1} \frac{(r+1)^{\gamma}-r^{\gamma}}{\gamma k^{\gamma}}}} \neq 0 .
$$

Hence, FP is invertible.

Theorem $9 I^{\gamma} F L(x)=\Psi F L(x)$ where

$$
\Psi=F P \Omega F P^{-1} .
$$

Proof Let

$$
I^{\gamma} F L(x)=\Psi F L(x) .
$$


From Eqs. (19) and (22)

$$
I^{\gamma} F L(x)=\Psi F P P(x)
$$

and

$$
\begin{aligned}
I^{\gamma} F L(x) & =F P I^{\gamma} P(x) \\
& =F P \Omega P(x) .
\end{aligned}
$$

Hence,

$$
\Psi F P P(x)=F P \Omega P(x),
$$

which implies that

$$
\Psi F P=F P \Omega
$$

or

$$
\Psi=F P \Omega F P^{-1} .
$$

Now, let $u \in C^{1}[0,1]$, then

$$
u(x)=\sum_{r=0}^{\infty} u_{r} F L_{r}(x)
$$

where

$$
u_{r}=(2 r+1) \gamma \int_{0}^{1} u(x) F L_{r}(x) x^{\gamma-1} d x
$$

Let

$$
U_{k}(x)=\sum_{r=0}^{k-1} u_{r} F L_{r}(x)=U^{T} F L(x)
$$

where

$$
U=\left(\begin{array}{c}
u_{0} \\
u_{1} \\
\vdots \\
u_{k-1}
\end{array}\right) .
$$

Thus,

$$
\mathfrak{f}_{r}(u(x))=U^{T} F L(x)=U^{T} F P P(x) .
$$


Using Theorem 3, we get

$$
\begin{aligned}
u(x)-\alpha & =I^{\gamma} U^{T} F L(x) \\
& =U^{T} I^{\gamma} F L(x) \\
& =U^{T} \Psi F L(x) \\
& =U^{T} \Psi F P P(x),
\end{aligned}
$$

which implies that

$$
u(x)=U^{T} \Psi F P P(x)+\alpha .
$$

Thus, Eq. (1) implies that

$$
U^{T} F P P(x)+\mu(x)\left(U^{T} \Psi F P P(x)+\alpha\right)+v(x)\left(U^{T} \Psi F P P(x)+\alpha\right)^{2}=\Xi F P P(x),
$$

where $\xi(x)=\Xi F P P(x)$. If

$$
U^{T} \Psi F P=\left(\begin{array}{llll}
\varkappa_{1} & \varkappa_{2} & \cdots & \varkappa_{k}
\end{array}\right)
$$

then

$$
\left(U^{T} \Psi F P P(x)+\alpha\right)^{2}=\left(\begin{array}{llll}
\varkappa_{1}^{2}+2 \varkappa_{1} \alpha & \varkappa_{2}^{2}+2 \varkappa_{2} \alpha & \cdots & \varkappa_{k}^{2}+2 \varkappa_{k} \alpha
\end{array}\right) P(x)+\alpha^{2} .
$$

Hence,

$$
\left(\digamma_{1}(U)+\mu(x) \digamma_{2}(U)+v(x) \digamma_{3}(U)\right) P(x)=\digamma_{4}(x)
$$

where

$$
\begin{aligned}
& \digamma_{1}(U)=U^{T} F P, \\
& \digamma_{2}(U)=U^{T} \Psi F P, \\
& \digamma_{3}(U)=\left(\varkappa_{1}^{2}+2 \varkappa_{1} \alpha \varkappa_{2}^{2}+2 \varkappa_{2} \alpha \cdots \varkappa_{k}^{2}+2 \varkappa_{k} \alpha\right), \\
& \digamma_{4}(x)=\Xi F P P(x)-\alpha \mu(x)-\alpha^{2} v(x) .
\end{aligned}
$$

To solve Eq. (48), we use the collocation points

$$
t_{r}=\frac{r+1}{k+1}, \quad r=0,1, \ldots, k-1
$$

Then we solve the generated nonlinear system to find $U$ using Mathematica.

\section{Numerical results}

We present two examples to show the efficiency of the proposed method. 
Example 1 Consider the following problem:

$$
\begin{aligned}
& £_{\frac{1}{2}}(u(x))+u(x)+u^{2}(x)=f(x), \quad 0<x<1, \\
& u(0)=1,
\end{aligned}
$$

where

$$
\begin{aligned}
f(x)= & x^{2}+1+\left(x^{2}+1\right)^{2}-4-4 x \\
& +\frac{8 \sqrt{x}(3+2 x)}{3 \sqrt{\pi}}+4 e^{x} \operatorname{Erfc}(\sqrt{x}) .
\end{aligned}
$$

The exact solution is

$$
u(x)=x^{2}+1
$$

Let $k=7$. Let $Q=\left\{p_{0}(x), p_{0}(x), \ldots, p_{6}(x)\right\}$ be the set of block pulse functions on $[0,1)$ where

$$
p_{r}(x)=\left\{\begin{array}{ll}
1, & \frac{r}{7} \leq x<\frac{r+1}{7}, \\
0, & \text { otherwise }
\end{array} \quad r=0,1, \ldots, 6\right.
$$

Let

$$
f(x)=\sum_{r=0}^{6} g_{r} p_{r}(x)=G^{T} P(x) .
$$

From Eq. (22), we have

$$
G=\left(\begin{array}{l}
2.03186 \\
2.21914 \\
2.59375 \\
3.16995 \\
3.97389 \\
5.04267 \\
6.42401
\end{array}\right), \quad P(x)=\left(\begin{array}{l}
p_{0}(x) \\
p_{1}(x) \\
p_{2}(x) \\
p_{3}(x) \\
p_{4}(x) \\
p_{5}(x) \\
p_{6}(x)
\end{array}\right) .
$$

From Eqs. (23) and (24), we have

$$
\Omega=\left(\begin{array}{ccccccc}
\frac{1}{2}+\frac{1}{\sqrt{6 \pi}} & \frac{1}{\sqrt{6 \pi}} & \frac{1}{\sqrt{6 \pi}} & \frac{1}{\sqrt{6 \pi}} & \frac{1}{\sqrt{6 \pi}} & \frac{1}{\sqrt{6 \pi}} & \frac{1}{\sqrt{6 \pi}} \\
\frac{1}{\sqrt{6 \pi}} & \frac{1}{2}+\frac{1}{\sqrt{6 \pi}} & \frac{1}{\sqrt{6 \pi}} & \frac{1}{\sqrt{6 \pi}} & \frac{1}{\sqrt{6 \pi}} & \frac{1}{\sqrt{6 \pi}} & \frac{1}{\sqrt{6 \pi}} \\
\frac{1}{\sqrt{6 \pi}} & \frac{1}{\sqrt{6 \pi}} & \frac{1}{2}+\frac{1}{\sqrt{6 \pi}} & \frac{1}{\sqrt{6 \pi}} & \frac{1}{\sqrt{6 \pi}} & \frac{1}{\sqrt{6 \pi}} & \frac{1}{\sqrt{6 \pi}} \\
\frac{1}{\sqrt{6 \pi}} & \frac{1}{\sqrt{6 \pi}} & \frac{1}{\sqrt{6 \pi}} & \frac{1}{2}+\frac{1}{\sqrt{6 \pi}} & \frac{1}{\sqrt{6 \pi}} & \frac{1}{\sqrt{6 \pi}} & \frac{1}{\sqrt{6 \pi}} \\
\frac{1}{\sqrt{6 \pi}} & \frac{1}{\sqrt{6 \pi}} & \frac{1}{\sqrt{6 \pi}} & \frac{1}{\sqrt{6 \pi}} & \frac{1}{2}+\frac{1}{\sqrt{6 \pi}} & \frac{1}{\sqrt{6 \pi}} & \frac{1}{\sqrt{6 \pi}} \\
\frac{1}{\sqrt{6 \pi}} & \frac{1}{\sqrt{6 \pi}} & \frac{1}{\sqrt{6 \pi}} & \frac{1}{\sqrt{6 \pi}} & \frac{1}{\sqrt{6 \pi}} & \frac{1}{2}+\frac{1}{\sqrt{6 \pi}} & \frac{1}{\sqrt{6 \pi}} \\
\frac{1}{\sqrt{6 \pi}} & \frac{1}{\sqrt{6 \pi}} & \frac{1}{\sqrt{6 \pi}} & \frac{1}{\sqrt{6 \pi}} & \frac{1}{\sqrt{6 \pi}} & \frac{1}{\sqrt{6 \pi}} & \frac{1}{2}+\frac{1}{\sqrt{6 \pi}}
\end{array}\right) .
$$


From Eq. (32), we have

$$
F P=\left(\begin{array}{ccccccc}
1 & 1 & 1 & 1 & 1 & 1 & 1 \\
-0.496 & -0.079 & 0.193 & 0.413 & 0.603 & 0.772 & 0.927 \\
-0.083 & -0.479 & -0.437 & -0.239 & 0.049 & 0.3977 & 0.791 \\
0.313 & 0.112 & -0.265 & -0.433 & -0.345 & 0.005 & 0.613 \\
-0.158 & 0.324 & 0.228 & -0.135 & -0.395 & -0.280 & 0.416 \\
-0.076 & -0.126 & 0.280 & 0.235 & -0.155 & -0.384 & 0.227 \\
0.125 & -0.228 & -0.083 & 0.281 & 0.146 & -0.309 & 0.068
\end{array}\right)
$$

It is easy to see that $F P$ is invertible, since $\operatorname{det}(F P)=1.4895 \times 10^{-3} \neq 0$. From Eq. (37), we have

$$
\begin{aligned}
\Psi & =F P \Omega F P^{-1} \\
& =\left(\begin{array}{ccccccc}
2.1 & -6.3 \times 10^{-4} & 2.1 \times 10^{-4} & 1.1 \times 10^{-2} & 4.2 \times 10^{-4} & -7.6 \times 10^{-4} & -8.2 \times 10^{-4} \\
0.5 & 0.50 & 6.6 \times 10^{-4} & 3.5 \times 10^{-3} & 5.4 \times 10^{-4} & -4.5 \times 10^{-4} & -2.3 \times 10^{-4} \\
4.5 \times 10^{-5} & -4.4 \times 10^{-5} & 0.50 & -6.4 \times 10^{-4} & 5.3 \times 10^{-5} & 2.3 \times 10^{-5} & 1.8 \times 10^{-5} \\
3.7 \times 10^{-5} & 2 \times 10^{-4} & -2.3 \times 10^{-5} & 0.50 & -1.4 \times 10^{-4} & 1.4 \times 10^{-4} & 4.4 \times 10^{-5} \\
-8 \times 10^{-5} & 1.1 \times 10^{-4} & -2.1 \times 10^{-4} & -3.5 \times 10^{-4} & 0.50 & 8.6 \times 10^{-5} & 9.9 \times 10^{-6} \\
1.4 \times 10^{-4} & -1.1 \times 10^{-4} & -9.7 \times 10^{-5} & 5.3 \times 10^{-4} & 6.3 \times 10^{-5} & 0.50 & -3.4 \times 10^{-5} \\
1.2 \times 10^{-5} & -1.4 \times 10^{-4} & 4.0 \times 10^{-5} & 6.2 \times 10^{-4} & 1.1 \times 10^{-4} & -1.1 \times 10^{-4} & 0.50
\end{array}\right) .
\end{aligned}
$$

By Eq. (47), we get

$$
U=\left(\begin{array}{c}
1.2 \\
0.4 \\
0.28571428569 \\
0.1 \\
0.01428571428 \\
0 \\
0
\end{array}\right)
$$

Thus,

$$
u_{7}(x)=\sum_{r=0}^{6} u_{r} F L_{r}(x)=1+x^{2}
$$

Hence, we get the exact solution.

Example 2 Consider the following problem:

$$
\begin{aligned}
& £_{\gamma}(u(x))+u(x)+u^{2}(x)=f(x), \quad 0<x<1,0<\gamma<1, \\
& u(0)=1,
\end{aligned}
$$

where

$$
f(x)=x^{\gamma+1}+x^{2 \gamma+2}+\frac{1+\gamma}{1-\gamma} \Gamma(1+\gamma) x^{1+\gamma} E_{\gamma, \gamma+2}\left(\frac{-\gamma x^{\gamma}}{1-\gamma}\right) .
$$


Table 1 Absolute errors

\begin{tabular}{ll}
\hline$\gamma$ & $R(\gamma)$ \\
\hline 0.3 & $1.21 * 10^{-13}$ \\
0.6 & $2.10 * 10^{-13}$ \\
0.9 & $2.32 * 10^{-13}$ \\
0.99 & $2.79 * 10^{-13}$ \\
\hline
\end{tabular}

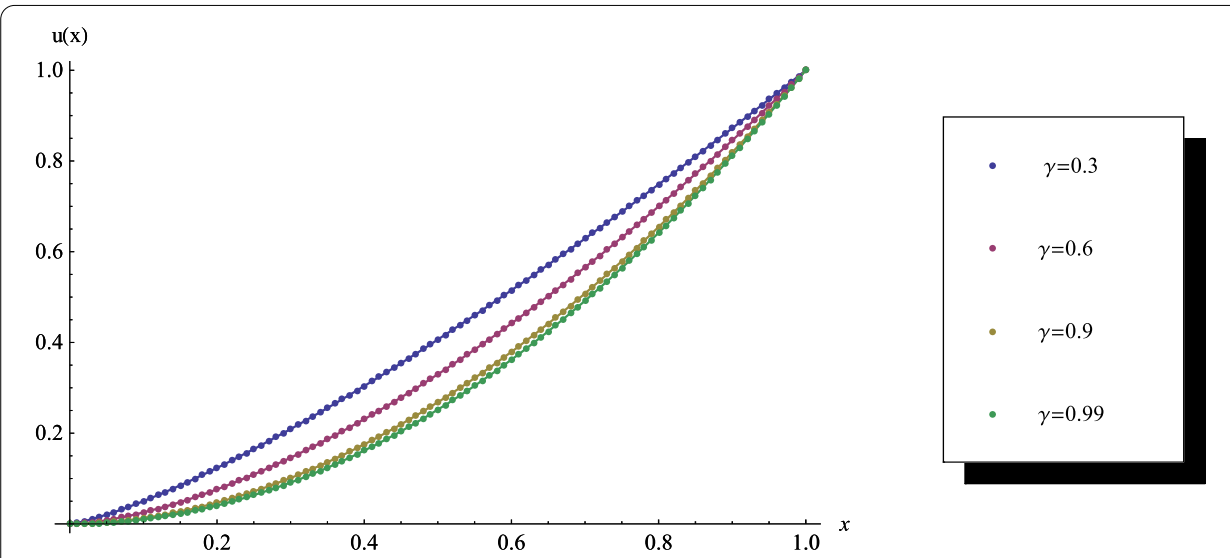

Figure 1 The exact and the approximate solutions for $\gamma=0.3,0.6,0.9$, and 0.99, dots: approximate solutions, joint lines: exact solutions

The exact solution is

$$
u(x)=x^{\gamma+1} .
$$

Let

$$
R(\gamma)=\max _{r=0,1, \ldots, 100}\left|u_{30}\left(\frac{r}{100}\right)-u\left(\frac{r}{100}\right)\right|
$$

Then the absolute errors for different choices of $\gamma$ are given in Table 1.

The graph of the exact and the approximate solutions for $\gamma=0.3,0.6,0.9$, and 0.99 are given in Fig. 1.

\section{Closing remarks}

In this article, we present a method to approximate the solution of FRE based on the ABFD in Caputo sense. The numerical method is based on the fractional operational matrix of the fractional derivative. We present two examples. In the first example, we get the exact solution. In the second one, the absolute error is of order $10^{-13}$. Results are given in Table 1. Figure 1 presents the agreement between the exact and the approximate solutions in Example 2 for different choices of $\gamma$. From the numerical results, we see that the proposed method gives accurate results. It is advisable to use it for other nonlinear fractional differential equations. 
Funding

Not applicable.

Ethics approval and consent to participate

Not applicable.

\section{Competing interests}

The authors declare that they have no competing interests.

\section{Consent for publication}

Not applicable.

\section{Authors' contributions}

The two authors contributed equally to the manuscript and typed, read, and approved the final manuscript.

\section{Author details}

'Department of Basic Sciences, King Saud University, Riyadh, Kingdom of Saudi Arabia. ${ }^{2}$ Department of Mathematical Sciences, UAE University, Al-Ain, United Arab Emirates.

\section{Publisher's Note}

Springer Nature remains neutral with regard to jurisdictional claims in published maps and institutional affiliations.

Received: 10 May 2019 Accepted: 2 July 2019 Published online: 16 July 2019

\section{References}

1. Kolwankar, K.M., Gangal, A.D.: Local fractional derivatives and fractal functions of several variables. In: Proceedings of the Conference on Fractals in Engineering, Archanon (1997)

2. Khalil, R., Horani, M.A., Yousef, A., Sababheh, M.: A new definition of fractional derivative. J. Comput. Appl. Math. 264 65-70 (2014)

3. Caputo, M., Fabrizio, M.: A new definition of fractional derivative without singular kernel. Prog. Fract. Differ. Appl. 1(2), 73-85 (2015)

4. Abdon, A., Dumitru, B.: New fractional derivatives with nonlocal and non-singular kernel: theory and application to heat transfer model. Therm. Sci. 20(2), 763-769 (2016)

5. Syam, M., Jaradat, H.M.: An accurate method for solving Riccati equation with fractional variable-order. J. Interpolat. Approx. Sci. Comput. 2018(1), Article ID jiasc-00120 (2018)

6. Kashkari, B., Syam, M.: Fractional-order Legendre operational matrix of fractional integration for solving the Riccati equation with fractional order. Appl. Math. Comput. 290, 281-291 (2016)

7. Zhang, Y: A finite difference method for fractional partial differential equation. Appl. Math. Comput. 215, 524-529 (2009). https://doi.org/10.1016/j.amc.2009.05.018

8. Akgul, A.: New reproducing kernel functions. Math. Probl. Eng. 2015, Article ID 158134 (2015)

9. Inc, M., Akgul, A., Kihicman, A.: Numerical solutions of the second-order one-dimensional telegraph equation based on reproducing kernel Hilbert space method. Abstr. Appl. Anal. 2013, Article ID 768963 (2013)

10. Inc, M., Akgul, A., Kihicman, A.: A novel method for solving KdV equation based on reproducing kernel Hilbert space method. Abstr. Appl. Anal. 2013, Article ID 578942 (2013)

11. Akgul, A., Khan, Y., Akgul, E., Baleanu, D., Al Qurashi, M.: Solutions of nonlinear systems by reproducing kernel method. J. Nonlinear Sci. Appl. 10, 4408-4417 (2017)

12. Boutarfa, B., Akgul, A., Inc, M.: New approach for the Fornberg-Whitham type equations. J. Comput. Appl. Math. 312, $13-26(2017)$

13. Akgul, A., Hashemi, M., Inc, M., Raheem, S.: Constructing two powerful methods to solve the Thomas-Fermi equation. Nonlinear Dyn. 87(2), 1435-1444 (2017)

14. Akgul, A., Inc, M., Kilicman, A., Baleanu, D.: A new approach for one-dimensional sine-Gordon equation. Adv. Differ. Equ. 2016, 8 (2016)

15. Akgul, A., Inc, M., Baleanu, D.: On solutions of variable-order fractional differential equations. Int. J. Optim. Control Theor. Appl. 7(1), 112-116 (2017)

16. Akgül, A., Kiliçman, A.: Solving delay differential equations by an accurate method with interpolation. Abstr. Appl. Anal. 2015, Article ID 676939 (2015)

17. Alquran, M., Al-Khaled, K., Sivasundaram, S., Jaradat, H.: Mathematical and numerical study of existence of bifurcations of the generalized fractional Burgers-Huxley equation. Nonlinear Stud. 24(1), 235-244 (2017)

18. Jaradat, I., Alquran, M., Al-Khaled, K.: An analytical study of physical models with inherited temporal and spatial memory. Eur. Phys. J. Plus 133, 162 (2018)

19. Jaradat, I., Al-Dolat, M., Al-Zoubi, K., Alquran, M.: Theory and applications of a more general form for fractional power series expansion. Chaos Solitons Fractals 108, 107-110 (2018)

20. Jaradat, I., Alquran, M., Al-Dolat, M.: Analytic solution of homogeneous time-invariant fractional IVP. Adv. Differ. Equ. 2018, $143(2018)$

21. Jaradat, I., Alquran, M., Abdalmohsen, R.: An analytical framework of $2 D$ diffusion, wave-like, telegraph, and Burgers' models with twofold Caputo derivatives ordering. Nonlinear Dyn. 93(4), 1911-1922 (2018)

22. Alquran, M., Jaradat, H., Syam, M.: Analytical solution of the time-fractional Phi-4 equation by using modified residual power series method. Nonlinear Dyn. 90(4), 2525-2529 (2017)

23. Alquran, M., Jaradat, I.: A novel scheme for solving Caputo time-fractional nonlinear equations: theory and application. Nonlinear Dyn. 91(4), 2389-2395 (2018)

24. Ali, M., Alquran, M., Jaradat, I.: Asymptotic-sequentially solution style for the generalized Caputo time-fractional Newell-Whitehead-Segel system. Adv. Differ. Equ. 2019, 70 (2019) 